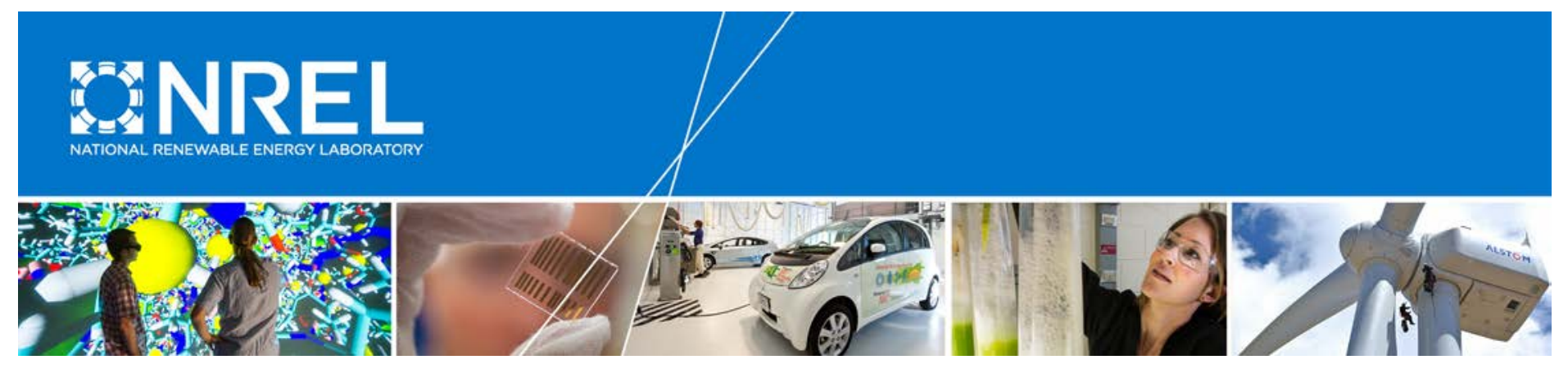

\title{
Three-Phase AC Optimal Power Flow Based Distribution Locational Marginal Price
}

\section{Preprint}

Rui Yang and Yingchen Zhang National Renewable Energy Laboratory

Presented at the IEEE Eighth Conference on Innovative Smart Grid Technologies (IEEE ISGT 2017)

Arlington, Virginia

April 23-26, 2017

(C) 2017 IEEE. Personal use of this material is permitted. Permission from IEEE must be obtained for all other uses, in any current or future media, including reprinting/republishing this material for advertising or promotional purposes, creating new collective works, for resale or redistribution to servers or lists, or reuse of any copyrighted component of this work in other works.

NREL is a national laboratory of the U.S. Department of Energy Office of Energy Efficiency \& Renewable Energy Operated by the Alliance for Sustainable Energy, LLC

This report is available at no cost from the National Renewable Energy Laboratory (NREL) at www.nrel.gov/publications.

\section{Conference Paper}

NREL/CP-5D00-68112

May 2017 


\section{NOTICE}

The submitted manuscript has been offered by an employee of the Alliance for Sustainable Energy, LLC (Alliance), a contractor of the US Government under Contract No. DE-AC36-08GO28308. Accordingly, the US Government and Alliance retain a nonexclusive royalty-free license to publish or reproduce the published form of this contribution, or allow others to do so, for US Government purposes.

This report was prepared as an account of work sponsored by an agency of the United States government. Neither the United States government nor any agency thereof, nor any of their employees, makes any warranty, express or implied, or assumes any legal liability or responsibility for the accuracy, completeness, or usefulness of any information, apparatus, product, or process disclosed, or represents that its use would not infringe privately owned rights. Reference herein to any specific commercial product, process, or service by trade name, trademark, manufacturer, or otherwise does not necessarily constitute or imply its endorsement, recommendation, or favoring by the United States government or any agency thereof. The views and opinions of authors expressed herein do not necessarily state or reflect those of the United States government or any agency thereof.

This report is available at no cost from the National Renewable Energy Laboratory (NREL) at www.nrel.gov/publications.

Available electronically at SciTech Connect http:/www.osti.gov/scitech

Available for a processing fee to U.S. Department of Energy and its contractors, in paper, from:

U.S. Department of Energy

Office of Scientific and Technical Information

P.O. Box 62

Oak Ridge, TN 37831-0062

OSTI http://www.osti.gov

Phone: 865.576.8401

Fax: 865.576.5728

Email: reports@osti.gov

Available for sale to the public, in paper, from:

U.S. Department of Commerce

National Technical Information Service

5301 Shawnee Road

Alexandria, VA 22312

NTIS http://www.ntis.gov

Phone: 800.553 .6847 or 703.605 .6000

Fax: 703.605.6900

Email: orders@ntis.gov 


\title{
Three-Phase AC Optimal Power Flow Based Distribution Locational Marginal Price
}

\author{
Rui Yang, Member, IEEE, and Yingchen Zhang, Senior Member, IEEE
}

\begin{abstract}
Designing market mechanisms for electricity distribution systems has been a hot topic due to the increased presence of smart loads and distributed energy resources in distribution systems. The distribution locational marginal pricing (DLMP) methodology is one of the real-time pricing methods to enable such market mechanisms and provide economic incentives to active market participants. Determining the DLMP is challenging due to high power losses, voltage volatility, and the phase imbalance in distribution systems. Existing DC optimal power flow (OPF) approaches are unable to model power losses and the reactive power, and single-phase AC OPF methods cannot capture the phase imbalance. To address these challenges, in this paper, a three-phase AC OPF based approach is developed to define and calculate the DLMP accurately. The DLMP is modeled as the marginal cost to serve an incremental unit of demand at a specific phase at a certain bus and is calculated using the Lagrange multipliers in the three-phase AC OPF formulation. Extensive case studies have been conducted to understand the impact of system losses and the phase imbalance on DLMPs as well as the potential benefits of flexible resources.
\end{abstract}

\section{INTRODUCTION}

Today's distribution systems have been experiencing a significant transformation due to an increasing amount of smart electric loads and distributed energy resources (DERs), such as electric vehicles, smart home appliances, rooftop photovoltaic systems, and energy storage. As more smart, flexible resources participate in distribution system operations, a market-based approach is necessary to manage future distribution systems efficiently and economically [1]. The distribution locational marginal price (DLMP) is a key component to enable power distribution markets and provide economic incentives to market participants.

The locational marginal price (LMP) represents the marginal cost to serve one incremental unit of demand at a specific location in electric power networks [2]-[4]. Traditionally, in transmission systems, LMP is technically calculated approximately by solving the direct current optimal power flow (DC OPF) problem [2], [3]. However, in distribution systems, power losses are usually high due to high $\mathrm{R} / \mathrm{X}$ ratio. In addition, reactive power modeling is needed as the voltage volatility is high. The DC OPF approach is unable to capture power losses and the reactive power in distribution systems. Therefore, it is unsuitable to be used to calculate the DLMP.

On the other hand, the single-phase alternating current optimal power flow (AC OPF) problem for the balanced system

The authors are with the National Renewable Energy Laboratory, Golden, CO 80401 USA (e-mail: rui.yang@nrel.gov; yingchen.zhang@nrel.gov). This work was supported by the U.S. Department of Energy under Contract No. DE-AC36-08GO28308 with the National Renewable Energy Laboratory. has also been proposed to calculate DLMPs [1], [5], [6]. For instance, a distribution system market mechanism based on convexification of single-phase AC OPF has been proposed in [1] to consider the voltage regulation and power losses. Another recent work [6] proposes to calculate the DLMP by approximating the single-phase AC OPF using linearized modeling of reactive power and power loss components. However, in many cases the distribution feeder configurations are untransposed, and the loads are unbalanced. Setting a uniform price for all three phases at the same bus does not provide the right incentives when large phase imbalance presents. Although the single-phase AC OPF approach is able to model the reactive power and power losses more accurately than the DC OPF approach, it does not consider the power imbalance among the three phases, which has a significant impact in distribution systems [7].

Therefore, the existing DC OPF and single-phase AC OPF approaches could not accurately model the key characteristics of distribution systems, and consequently the DLMPs derived based on these approaches may not be accurate enough to fully incentivize the market participation of flexible resources. In this paper, a three-phase AC OPF problem is developed to define and calculate DLMPs. The three-phase AC OPF problem optimizes the active and the reactive power simultaneously while taking into account the unbalanced nature of distribution systems accurately. This full-blown AC OPF approach provides a solid foundation to systematically derive, understand, and analyze DLMPs.

In this paper, a three-phase unbalanced AC OPF model of the power distribution networks is first built. Based on the three-phase AC OPF formulation, the DLMP is defined as the marginal cost to serve an incremental unit of demand at a specific phase at a certain bus. Hence, the DLMP can be derived and calculated using the Lagrange multipliers associated with the corresponding per-phase power balance constraints. Extensive case studies have been conducted to understand how the system losses and the phase imbalance impact the DLMPs and the potential benefits flexible resources may bring.

The rest of the paper is organized as follows: The threephase modeling of distribution systems is first introduced in Section II. In Section III, the developed three-phase AC OPF problem is presented, followed by the derivation of the DLMPs. The case study results on a real distribution system are given in Section IV. Finally, Section V concludes the paper and discusses future research directions and open questions. 


\section{Distribution System Modeling}

In this section, the three-phase models of the distribution system components are first explained, and then the distribution AC power flow equations are derived.

For a branch connecting buses $i$ and $j$ in the distribution system, let $\mathcal{P}_{i j} \subseteq\left\{a_{i j}, b_{i j}, c_{i j}\right\}$ denote the set of phases to which this branch is connected and $I_{i j}, I_{j i}$ represent the vectors of line currents $I_{i j}^{\phi}$ from bus $i$ to $j$ and $I_{j i}^{\phi}$ from $j$ to $i$ in all phases $\phi \in \mathcal{P}_{i j}$. The branch currents can be written as [8]:

$$
\left[\begin{array}{c}
I_{i j} \\
I_{j i}
\end{array}\right]=Y_{i j}^{B R} \cdot\left[\begin{array}{c}
V_{i} \\
V_{j}
\end{array}\right]=\left[\begin{array}{cc}
Y_{i j}^{i i} & Y_{i j}^{i j} \\
Y_{i j}^{j i} & Y_{i j}^{j j}
\end{array}\right] \cdot\left[\begin{array}{c}
V_{i} \\
V_{j}
\end{array}\right]
$$

where $V_{i}$ and $V_{j}$ are the vectors of voltages at buses $i$ and $j$ in all phases. The matrix $Y_{i j}^{B R}$ is the branch admittance matrix which maps the voltages at both ends to the branch currents.

The branch admittance matrix can be used to model the series components, such as distribution lines and transformers. For a distribution line connecting buses $i$ and $j$, the standard $\pi$-model is employed here [7] with matrices $Z_{l, i j}$ and $Y_{s, i j}$ representing the series impedance and the shunt admittance matrices of this line, respectively. The branch admittance matrix of branch $i j$ is as follows:

$$
Y_{i j}^{B R}=\left[\begin{array}{cc}
Z_{l, i j}^{-1}+\frac{1}{2} Y_{s, i j} & -Z_{l, i j}^{-1} \\
-Z_{l, i j}^{-1} & Z_{l, i j}^{-1}+\frac{1}{2} Y_{s, i j}
\end{array}\right]
$$

For transformers, based on the connection type and parameters of both the primary and secondary sides, branch admittance matrices can also be determined accordingly [8].

With the branch components modeled using the branch admittance matrices, the branch current $I_{i j}$ is:

$$
I_{i j}=Y_{i j}^{i i} \cdot V_{i}+Y_{i j}^{i j} \cdot V_{j}
$$

The total current flowing out of bus $i$ in phase $\phi$ is:

$$
I_{i}^{\phi}=\sum_{j \in \mathcal{N}_{i}}\left(Y_{i j}^{i i} \cdot V_{i}+Y_{i j}^{i j} \cdot V_{j}\right)_{\{\phi\}}
$$

where $\mathcal{N}_{i}$ is the set of buses which are connected to bus $i$.

Therefore, the complex power balance equation at bus $i$ phase $\phi$ can be expressed as:

$$
V_{i}^{\phi} \cdot\left(I_{i}^{\phi}\right)^{*}=P_{G, i}^{\phi}-P_{L, i}^{\phi}+\mathbf{j}\left(Q_{G, i}^{\phi}-Q_{L, i}^{\phi}\right)
$$

where $P_{G, i}^{\phi}, Q_{G, i}^{\phi}$ are the active and reactive power generation and $P_{L, i}^{\phi}, Q_{L, i}^{\phi}$ the active and reactive demand at bus $i$ phase $\phi$.

In this paper, only the single-phase and three-phase wyeconnected generators and loads are considered, whose power generation/consumption in each phase can be modeled independently. Further investigation is needed to integrate deltaconnected generators and loads into the considered three-phase AC OPF problem.

\section{DLMP FORMULATION}

The LMP at a certain location represents the incremental cost to supply an extra unit of load at this location. Traditionally, the DC OPF problem is used to determine the LMPs in the transmission level. In order to accurately determine the DLMPs, the unique characteristics of distribution systems should be modeled by employing the three-phase AC OPF problem. The formulation of the considered three-phase $\mathrm{AC}$ OPF problem is as follows:

$$
\begin{array}{ll}
\min _{P_{G, k}^{\phi}} & \sum_{k \in \mathcal{N}_{G}} \sum_{\phi \in \mathcal{P}_{G, k}} C_{i}\left(P_{G, k}^{\phi}\right) \\
\text { s.t. } & P_{G, i}^{\phi}-P_{L, i}^{\phi}=\Re\left\{V_{i}^{\phi} \cdot\left(I_{i}^{\phi}\right)^{*}\right\} \\
& Q_{G, i}^{\phi}-Q_{L, i}^{\phi}=\Im\left\{V_{i}^{\phi} \cdot\left(I_{i}^{\phi}\right)^{*}\right\} \\
& \underline{P}_{G, k}^{\phi} \leq P_{G, k}^{\phi} \leq \bar{P}_{G, k}^{\phi} \\
& \underline{Q}_{G, k}^{\phi} \leq Q_{G, k}^{\phi} \leq \bar{Q}_{G, k}^{\phi} \\
& -\bar{P}_{i j}^{\phi} \leq P_{i j}^{\phi} \leq \bar{P}_{i j}^{\phi} \\
& \underline{V}_{i}^{\phi} \leq\left|V_{i}^{\phi}\right| \leq \bar{V}_{i}^{\phi}
\end{array}
$$

where $\mathcal{N}_{G}$ denotes the set of buses which have generators connected and $\mathcal{P}_{G, k}$ the set of phases to which the generator at bus $k$ is connected. $\Re\{\cdot\}$ and $\Im\{\cdot\}$ denote the real and the imaginary parts of a complex number, respectively. $P_{G, k}^{\phi}$ and $Q_{G, k}^{\phi}$ represent the active and reactive power output of the generator at bus $k$ phase $\phi, \underline{P}_{G, k}^{\phi}, \bar{P}_{G, k}^{\phi}$ the corresponding lower and upper limits of the active power generation, and $\underline{Q}_{G, k}^{\phi}, \bar{Q}_{G, k}^{\phi}$ the lower and upper limits of the reactive power generation. $P_{i j}^{\phi}$ corresponds to the active power flow in branch ij phase $\phi$ with $\bar{P}_{i j}^{\phi}$ as its upper bound. $\left|V_{i}^{\phi}\right|$ corresponds to the voltage magnitude at bus $i$ phase $\phi$, and $\underline{V}_{i}^{\phi}, \bar{V}_{i}^{\phi}$ the associated lower and upper bounds.

In the considered $\mathrm{AC}$ OPF problem, the control variables are the active and reactive power output of the generators. Here, both the conventional generators such as diesel generators as well as distributed generators (DGs) such as smart inverter connected distributed-generation photovoltaic systems are considered. The objective function is to minimize the total generation cost of the active power. The equality constraints (7) and (8) correspond to the active and reactive power balance equations at bus $i$ phase $\phi$, respectively. The inequality constraints include the active and reactive power generation limits (9) and (10) as well as the operational constraints on line flows (11) and voltages (12). By solving the formulated AC OPF problem, the optimal active and reactive generation dispatch is determined such that the total generation cost of the active power is minimized while all the operational constraints are satisfied.

For simplicity, let $u$ denote the vector of all control variables and $x$ the vector of all state variables, including the voltage magnitude and angle at every bus and phase. Define $h(x, u) \leq 0$ to represent the inequality constraints in (9)-(12). The Lagrangian function of the formulated AC OPF problem 
can be written as:

$$
\begin{aligned}
& L(x, u, \lambda, \nu, \mu)=\sum_{k \in \mathcal{N}_{\mathcal{G}}} \sum_{\phi \in \mathcal{P}_{G, k}} C_{i}\left(P_{G, k}^{\phi}\right) \\
& -\sum_{i \in \mathcal{N}} \sum_{\phi \in \mathcal{P}_{i}} \lambda_{i}^{\phi} \cdot\left(P_{G, i}^{\phi}-P_{L, i}^{\phi}-\Re\left\{V_{i}^{\phi} \cdot\left(I_{i}^{\phi}\right)^{*}\right\}\right) \\
& -\sum_{i \in \mathcal{N}} \sum_{\phi \in \mathcal{P}_{i}} \nu_{i}^{\phi} \cdot\left(Q_{G, i}^{\phi}-Q_{L, i}^{\phi}-\Im\left\{V_{i}^{\phi} \cdot\left(I_{i}^{\phi}\right)^{*}\right\}\right) \\
& +\sum_{m \in \mathcal{H}} \mu_{m} \cdot h_{m}(x, u)
\end{aligned}
$$

where $\mathcal{N}$ represents the set of all buses in the system, $\mathcal{P}_{i}$ the set of phases at bus $i$, and $\mathcal{H}$ the set of inequality constraints. $\lambda_{i}^{\phi}$ and $\nu_{i}^{\phi}$ are the Lagrange multipliers corresponding to the active power balance equation (7) and the reactive power balance equation (8) at bus $i$ phase $\phi$, respectively. $\mu_{m}$ is the Lagrange multiplier associated with the inequality constraint $h_{m}(x, u) \leq 0$.

In the following, DLMPs are derived using a similar approach as deriving the LMPs in the transmission system based on the AC OPF problem [9].

Assume that the considered AC OPF problem has an optimal solution $\left(x^{*}, u^{*}\right)$. As the DLMP represents the marginal cost to supply the next increment of load, the DLMP can be calculated as:

$$
D L M P_{i}^{\phi}=\left.\frac{\partial f}{\partial P_{L, i}^{\phi}}\right|_{x^{*}, u^{*}}=\left.\frac{\partial L}{\partial P_{L, i}^{\phi}}\right|_{x^{*}, u^{*}}=\lambda_{i}^{\phi}
$$

where $f$ represents the objective function (6) of the $\mathrm{AC}$ OPF problem. The DLMP at bus $i$ phase $\phi$ is the Lagrange multiplier $\lambda_{i}^{\phi}$ associated with the active power balance equation at this bus and phase, which represents the incremental generation cost if the load at bus $i$ phase $\phi$ increases by one unit. Hence, by solving the three-phase AC OPF problem, the resulting DLMPs could be different not only with respect to different locations but also different phases. This is due to the unbalanced nature of distribution systems.

In this paper, the cost of reactive power generation is not explicitly considered in the objective function. However, by incorporating the operational constraints (12) of voltage magnitudes in the formulated AC OPF problem, the impact of the reactive power and voltages on DLMPs has already been considered implicitly.

\section{CAse Study}

The case study of the three-phase AC OPF based DLMPs has been conducted on a 60-bus system based on a real campus grid. In this section, the test system is first presented, followed by the detailed analysis of the three-phase AC OPF based DLMPs under two different cases.

\section{A. Simulation Setup}

The campus distribution grid of the University of Denver (DU) is used as the test system in this paper. The system topology is shown in Fig. 1 [10]. The campus grid is connected to the utility grid at buses 1, 38, and 51; and the other 57 buses represent the buildings on campus. Due to the lack of

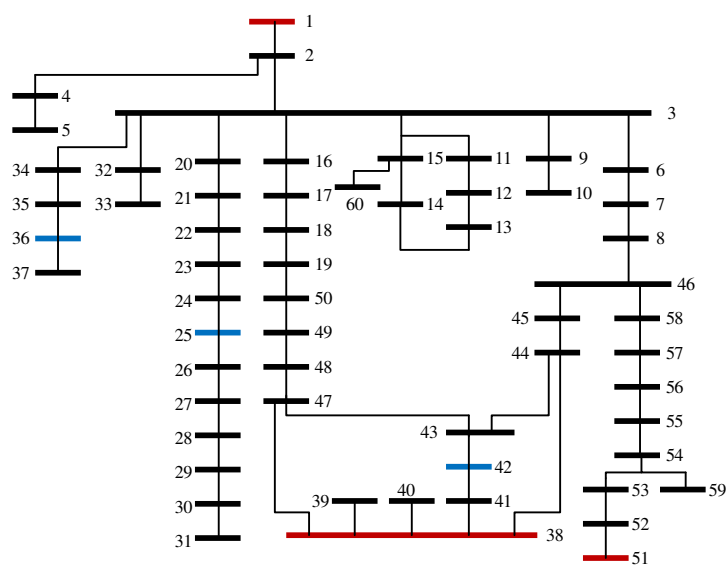

Fig. 1. DU campus grid-based 60-bus test system.

TABLE I

SUMMARY OF UNBALANCED LOAD DATA

\begin{tabular}{cc|cc|cc}
\hline Phase & No & $P(\mathrm{~kW})$ & $P(\%)$ & $Q(\mathrm{kVar})$ & $Q(\%)$ \\
\hline $\mathrm{A}$ & 26 & 708.9594 & 37.94 & 288.0956 & 44.81 \\
\hline $\mathrm{B}$ & 17 & 541.7142 & 28.99 & 157.8817 & 24.55 \\
\hline $\mathrm{C}$ & 20 & 618.0264 & 33.07 & 197.0224 & 30.64 \\
\hline Total & 57 & 1868.7 & 100 & 643 & 100 \\
\hline
\end{tabular}

information about the campus grid parameters, some typical line parameters from IEEE test feeders are used in the DU system. Synthetic load data from some real utility systems has been used to create a realistic unbalanced load profile for the test system, with the total active and reactive power consumption as $1868.7 \mathrm{~kW}$ and $643 \mathrm{kVar}$, respectively. The unbalanced load data is depicted in Fig. 2, and the summary of the per-phase load is provided in Table I. The column 'No' gives the number of loads which are connected to each phase, columns ' $P(\mathrm{~kW})$ ' and ' $P(\%)$ ' represent the total active power consumption of each phase and the percentage of the per-phase active power consumption corresponding to the total active power consumption, while ' $Q$ (kVar)' and ' $Q$ (\%)' represent the corresponding information of the perphase reactive power consumption. There are three three-phase wye-connected loads in the system, while the rest are singlephase loads. In the considered load scenario, phase A has the largest total demand, while phase B has the smallest. The lower and upper limits for the voltage magnitudes are 0.95 p.u. and 1.05 p.u., respectively. In the considered load scenario, no operational constraints of line flows, voltage magnitudes, and reactive power generation limits are binding.

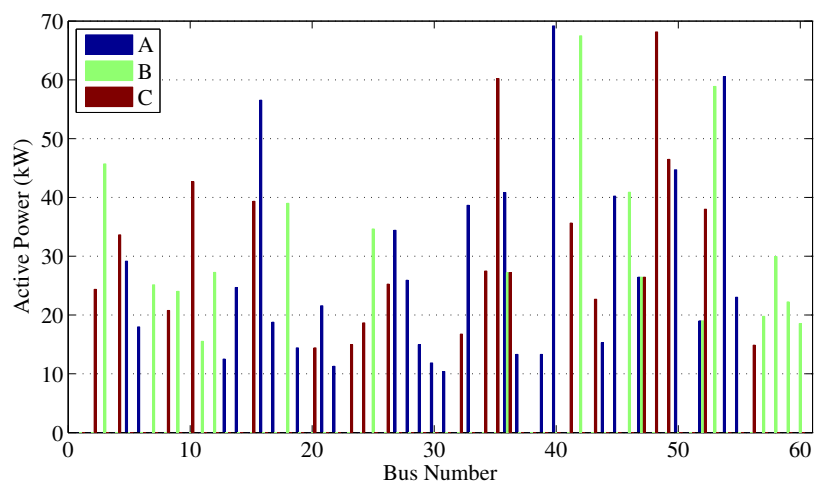

Fig. 2. Unbalanced load data of the test system. 


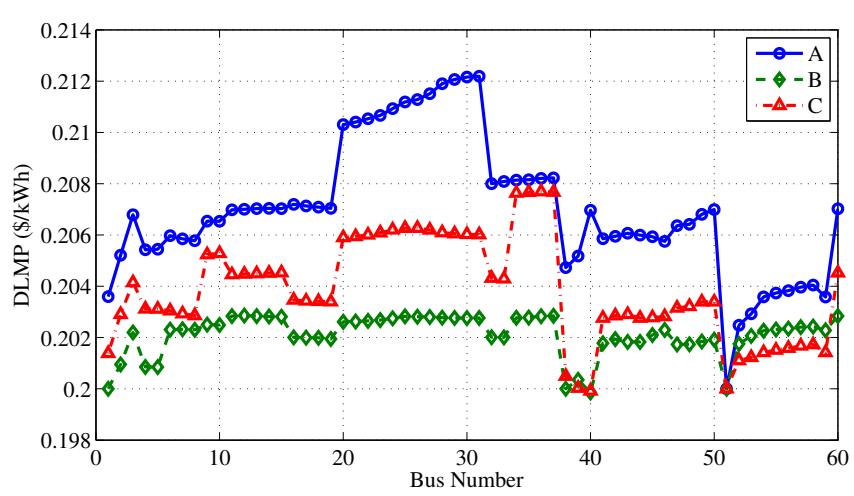

Fig. 3. DLMPs in Case 1.

TABLE II

Generation Results in CASE 1

\begin{tabular}{c|c|c|c|c|c|c}
\hline \multirow{2}{*}{ Bus } & \multicolumn{2}{|c|}{ A } & \multicolumn{2}{c|}{ B } & \multicolumn{2}{c}{ C } \\
\cline { 2 - 7 } & $\begin{array}{c}\text { Gen. } \\
(\mathrm{kW})\end{array}$ & $\begin{array}{c}\text { DLMP } \\
(\$ / \mathrm{kWh})\end{array}$ & $\begin{array}{c}\text { Gen. } \\
(\mathrm{kW})\end{array}$ & $\begin{array}{c}\text { DLMP } \\
(\$ / \mathrm{kWh})\end{array}$ & $\begin{array}{c}\text { Gen. } \\
(\mathrm{kW})\end{array}$ & $\begin{array}{c}\text { DLMP } \\
(\$ / \mathrm{kWh})\end{array}$ \\
\hline 1 & 250 & 0.2036 & 183.4010 & 0.2 & 250 & 0.2014 \\
\hline 38 & 250 & 0.2047 & 203.4780 & 0.2 & 250 & 0.2005 \\
\hline 51 & 216.0532 & 0.2 & 157.7956 & 0.2 & 124.9190 & 0.2 \\
\hline
\end{tabular}

\section{B. Case 1: Conventional Generators}

The first case considered in this paper uses three conventional generators to represent the connection of the campus grid to the utility grid, i.e., the three-phase wye-connected conventional generators at buses 1,38 , and 51. Each conventional generator would like to sell active power at a price of $\$ 0.2 / \mathrm{kWh}$ up to $250 \mathrm{~kW}$ per phase. Hence, the objective function of the formulated AC OPF problem is a linear function of the generation output, which is bounded by $[0$, $250 \mathrm{~kW}]$ per phase. By solving the formulated three-phase AC OPF problem, the optimal generation dispatch and DLMPs are determined. Table II shows the optimal active power output and the price received of each generator. Fig. 3 illustrates the DLMPs in the system. At a glance, we can conclude that the DLMPs at different buses and different phases vary significantly, which are able to create locational incentives for DERs.

As shown in Table II, since the generator at bus 51 is not producing at its maximum capacity in all three phases, it will be able to supply the next increment of load in any phase at this bus by increasing the generation output in the same phase accordingly. Consequently, the DLMPs at this bus in all three phases are the same as the marginal generation cost of the generator at this bus, which is $\$ 0.2 / \mathrm{kWh}$. The other two generators at buses 1 and 38 are producing at their maximum capacities in phases A and C. Hence, if the load at these two buses in phases $\mathrm{A}$ and $\mathrm{C}$ slightly increases, the generation output at the corresponding bus and phase could not be increased to accommodate any additional demand, resulting in higher DLMPs at these two buses in phases A and C than the marginal generation cost of generators.

DLMPs as depicted in Fig. 3 are different across the whole system at different buses and different phases due to the power losses and unbalanced system parameters and loads. Phase A has the largest active power consumption compared to the other two phases, and the active power losses are also larger in phase A than the losses in the other two phases. The total

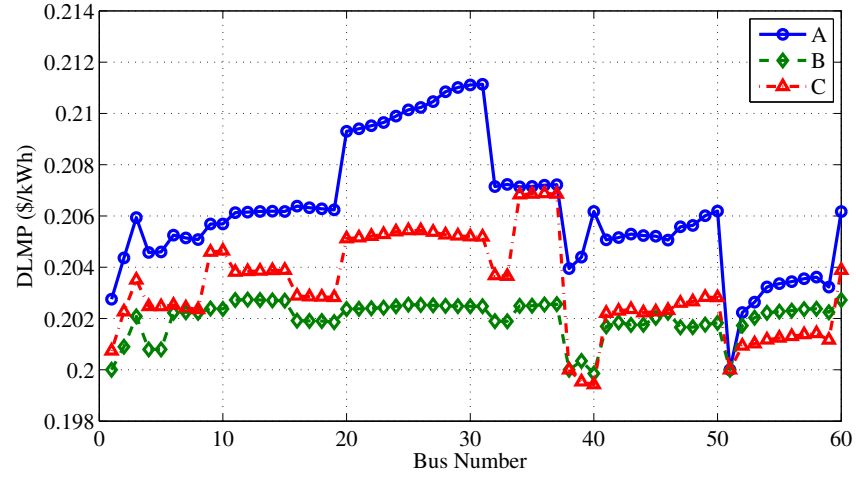

Fig. 4. DLMPs in Case 2.

active power losses in phases $\mathrm{A}, \mathrm{B}$, and $\mathrm{C}$ are $7.0938 \mathrm{~kW}$, $2.9603 \mathrm{~kW}$, and $6.8926 \mathrm{~kW}$, respectively. Consequently, the DLMPs in phase A are larger than the DLMPs in phases B and C. Especially, the DLMPs at buses 20 to 31 in phase A are significantly larger than the DLMPs in the rest of the system. The reason for that lies in the system structure and the determined optimal generation output. The lateral from buses 20 to 31 has significantly higher active power consumption than that in phases $\mathrm{B}$ and $\mathrm{C}$ and is directly connected to the generator at bus 1 through bus 2 while weakly connected to generators at buses 38 and 51 through the slightly meshed system. If the active power demand in this lateral in phase A slightly increases, the most cost-effective way to supply the additional demand is increasing the power generation at bus 1 phase A. However, the generation output at bus 1 phase A has already reached its limit. Hence, the power generation at other buses and phases needs to be increased, rendering higher active power losses and thereby larger DLMPs. If a DG is installed in this lateral, it will be able to reduce the active power losses by supplying the demand at nearby buses, resulting in lower DLMPs in this lateral, which will be demonstrated in Case 2.

Since phase B has the lowest load, the DLMPs in phase B are closer to the marginal generation cost of the generators, i.e., $\$ 0.2 / \mathrm{kWh}$. An interesting observation is that the DLMPs in phase $\mathrm{C}$ are higher than those in phase $\mathrm{B}$ in most parts of the system with exception at buses 52 to 59 . In general, the phase $\mathrm{C}$ active power consumption is larger than the phase $\mathrm{B}$ consumption in most parts of the system, resulting in higher DLMPs in phase C. However, at buses 52 to 59, the active power demand in phase B is larger than that in phase C, leading to larger active power losses and larger DLMPs.

\section{Case 2: Conventional Generators + DGs}

In Case 2, three additional DGs (three-phase wyeconnected) are added at buses 25,36 , and 42 , each of which would like to sell active power at a price of $\$ 0.04 / \mathrm{kWh}$ up to $6 \mathrm{~kW}$ per phase. The optimal generation dispatch by solving the three-phase AC OPF problem is summarized in Table III, and the DLMPs in the system are depicted in Fig. 4.

With three additional DGs in the system which have much lower bid prices than those of conventional generators, all three DGs are dispatched to generate active power at their maximum capacities in every phase. As a result, the generation output 
TABLE III

GENERATION RESULTS IN CASE 2

\begin{tabular}{c|c|c|c|c|c|c}
\hline \multirow{2}{*}{ Bus } & \multicolumn{2}{|c|}{ A } & \multicolumn{2}{c|}{ B } & \multicolumn{2}{c}{ C } \\
\cline { 2 - 7 } & $\begin{array}{c}\text { Gen. } \\
(\mathrm{kW})\end{array}$ & $\begin{array}{c}\text { DLMP } \\
(\$ / \mathrm{kWh})\end{array}$ & $\begin{array}{c}\text { Gen. } \\
(\mathrm{kW})\end{array}$ & $\begin{array}{c}\text { DLMP } \\
(\$ / \mathrm{kWh})\end{array}$ & $\begin{array}{c}\text { Gen. } \\
(\mathrm{kW})\end{array}$ & $\begin{array}{c}\text { DLMP } \\
(\$ / \mathrm{kWh})\end{array}$ \\
\hline 1 & 250 & 0.2028 & 177.0610 & 0.2 & 250 & 0.2007 \\
\hline 38 & 250 & 0.2039 & 196.2287 & 0.2 & 245.5294 & 0.2 \\
\hline 51 & 197.4412 & 0.2 & 153.1246 & 0.2 & 110.8578 & 0.2 \\
\hline 25 & 6 & 0.2101 & 6 & 0.2025 & 6 & 0.2054 \\
\hline 36 & 6 & 0.2072 & 6 & 0.2025 & 6 & 0.2069 \\
\hline 42 & 6 & 0.2052 & 6 & 0.2019 & 6 & 0.2023 \\
\hline
\end{tabular}

of three conventional generators is reduced. In particular, the generator at bus 38 is no longer producing at its maximum capacity in phase $\mathrm{C}$, hence the DLMP at bus 38 phase $\mathrm{C}$ is reduced to $\$ 0.2 / \mathrm{kWh}$, which equals the marginal generation cost of the conventional generator at this bus.

Compared to Case 1 without any DG in the system, the total active power losses in phases $\mathrm{A}, \mathrm{B}$, and $\mathrm{C}$ are reduced to $6.4817 \mathrm{~kW}, 2.7002 \mathrm{~kW}$, and $6.3608 \mathrm{~kW}$, respectively, as a result of DGs being able to supply part of the demand within their local areas. Therefore, the DLMPs in Case 2 are smaller than or equal to the DLMPs in Case 1 at every bus and phase. Significant reductions in DLMPs are seen in the lateral from buses 20 to 31 in phase A, in which the DLMPs are the highest throughout the whole system. The reason for that is the DG at bus 25 supplies some demand in the lateral and consequently reduces the active power losses. This demonstrates the potential benefits which DGs may bring in distribution systems.

As phase imbalance of system parameters and loads leads to the phase difference of DLMPs, the DLMP at bus 40 phase $\mathrm{C}$ shown in Fig. 4 is an interesting example demonstrating how the system imbalance would impact the DLMPs. The DLMP at bus 40 phase $\mathrm{C}$ is $\$ 0.1995 / \mathrm{kWh}$, which is even lower than the marginal generation cost of the conventional generators- $\$ 0.2 / \mathrm{kWh}$. Since the DGs are already generating at their maximum capacities in all three phases, if the load slightly increases at bus 40 phase $\mathrm{C}$, the generation output from conventional generators needs to be increased to accommodate the additional demand. The DLMP at bus 40 phase $\mathrm{C}$ lower than $\$ 0.2 / \mathrm{kWh}$ indicates that less increase in the generation output than the increase in the load is needed. For instance, if the active power consumption at bus 40 phase $\mathrm{C}$ increases by $0.01 \mathrm{~kW}$, the increased active power generation from the conventional generators is less than $0.01 \mathrm{~kW}$, which is caused by the extreme phase imbalance of the voltage magnitudes at bus 40. If the load in phase $\mathrm{C}$ increases, the phase imbalance of voltage magnitudes at bus 40 would be reduced, resulting in less active power losses and less increase in the generation output.

In summary, as shown in the cases without and with DGs in the system, the three-phase AC OPF based DLMPs are different with respect to different locations and different phases due to the distribution system losses and phase imbalance. Hence, AC OPF based DLMPs represent not only the locational benefits but also the phase benefits that DERs or demand response may bring, which will be essential to support a distribution-level marketplace.

\section{CONCLUSION AND FUtURE WORK}

In this paper, a three-phase AC OPF based approach is developed to define and calculate DLMPs. By formulating the three-phase AC OPF problem, DLMPs are derived using the Lagrange multipliers of the corresponding per-phase power balance constraints. As shown in the case studies, three-phase AC OPF based DLMPs can be different at different locations and phases due to the phase imbalance and power losses. We also demonstrate from the case studies that potential market participants in distribution systems can benefit from the DLMP-based markets while driving down the cost of the entire distribution system.

Future research directions include: First, we need to study how market mechanisms can be established using the threephase AC OPF based DLMP to provide the right incentives. Open questions remain on how employing such DLMPs leads to more efficient power delivery in distribution systems. On the other hand, how market participants react to such threephase AC OPF based DLMPs and how they make optimal decisions also need to be further investigated.

Secondly, the impact of the reactive power on DLMPs has not yet been well understood. We need to study how voltage constraints contribute to DLMPs and how reactive power components interact with the phase imbalance and system losses. Further case studies are needed to quantify the significance of each component in DLMPs.

Finally, efficient computational approaches to calculate and approximate DLMPs are needed to bring DLMPs to practice. While the three-phase AC OPF provides a comprehensive and accurate model of distribution systems, solving such problems in large-scale systems is computationally intensive. Therefore, we need to develop computationally efficient methods to calculate DLMPs.

\section{REFERENCES}

[1] N. Li, "A market mechanism for electric distribution networks," in 2015 54th IEEE Conference on Decision and Control (CDC). IEEE, 2015, pp. 2276-2282.

[2] S. S. Oren, P. T. Spiller, P. Varaiya, and F. Wu, "Nodal prices and transmission rights: A critical appraisal," The Electricity Journal, vol. 8, no. 3, pp. 24-35, 1995.

[3] E. Litvinov, "Design and operation of the locational marginal pricesbased electricity markets," IET generation, transmission \& distribution, vol. 4, no. 2, pp. 315-323, 2010.

[4] E. Litvinov, T. Zheng, G. Rosenwald, and P. Shamsollahi, "Marginal loss modeling in LMP calculation," IEEE transactions on Power Systems, vol. 19, no. 2, pp. 880-888, 2004.

[5] R. Li, Q. Wu, and S. S. Oren, "Distribution locational marginal pricing for optimal electric vehicle charging management," IEEE Transactions on Power Systems, vol. 29, no. 1, pp. 203-211, 2014.

[6] H. Yuan, F. Li, Y. Wei, and J. Zhu, "Novel linearized power flow and linearized opf models for active distribution networks with application in distribution LMP," IEEE Transactions on Smart Grid, 2016.

[7] W. H. Kersting, Distribution system modeling and analysis. CRC press, 2012.

[8] R. D. Zimmerman, "Comprehensive distribution power flow: modeling, formulation, solution algorithms and analysis," Ph.D. dissertation, Cornell University, 1995.

[9] H. Liu, L. Tesfatsion, and A. A. Chowdhury, "Locational marginal pricing basics for restructured wholesale power markets," in IEEE Power Energy Society General Meeting, Calgary, AB, Canada, July 2009.

[10] J. Hao, Y. Gu, Y. Zhang, J. J. Zhang, and D. W. Gao, "Locational marginal pricing in the campus power system at the power distribution level," in IEEE Power Energy Society General Meeting, Boston, MA, USA, July 2016. 